PEREIN ON THE HYDRIDE OF ACETO-SALICYL.

XXIII.-On the Hydride of Aceto-Salicyl.

By W. H. Perkin, F.R.S.

IN some considerations on the formation of coumarin by means of the hydride of sodium-salicyl and acetic anhydride, which I had the honour of laying before the Society only a short time since,* I mentioned that the formation of this body appeared to be the result of two distinct reactions-the first consisting in the replacement of an equivalent of hydrogen in the hydride of salicyl by acetyl-the second in the removal of a molecule of water from this acetylic derivative. I also stated that I had succeeded in obtaining the body resulting from the first reaction, and which I termed the hydride of aceto-salicyl. The present

* Page 60 of this volume. 
communication is intended to give an account of the preparation and properties of this substance, and to show under what circumstances it may be converted into coumarin.

\section{Hydride of Acetosalicyl.}

To prepare this body, acetic anhydride is added to powdered anhydrous hydride of sodium-salicyl suspended in pure dry ether-the reagents being employed in equivalent quantities;chemical action then gradually sets in, the sodium compound at the same time losing its yellow colour. After standing for twenty-four hours, the ethereal liquid is filtered off from the acetate of sodium which has formed, and evaporated upon the water-bath. In this manner an oily body is obtained, which gradually solidifies to a crystalline cake upon cooling. This is purified by pressure between bibulous paper, and subsequent crystallisation from alcohol. Three combustions of this body gave the following numbers:-

$$
\begin{aligned}
& \text { I. }-2977 \text { of substance gave } \\
& \cdot 7161 \text { of } \mathrm{CO}_{2} \text {, and } \\
& \cdot 1441 \text { of } \mathrm{H}_{2} \mathrm{O} \text {. } \\
& \text { II. } \cdot 2286 \text { of substance gave } \\
& .5499 \text { of } \mathrm{CO}_{2} \text {, and } \\
& \cdot 1051 \text { of } \mathrm{H}_{2} \mathrm{O} \text {. } \\
& \text { III. } \cdot 2588 \text { of substance gave } \\
& \cdot 6240 \text { of } \mathrm{CO}_{2} \text {, and } \\
& \cdot 1171 \text { of } \mathrm{H}_{2} \mathrm{O} \text {. }
\end{aligned}
$$

These numbers give percentages agreeing with the formula :

$$
\mathrm{C}_{9} \mathrm{H}_{8} \mathrm{O}_{3} \text {, }
$$

as the following comparisons will show :-

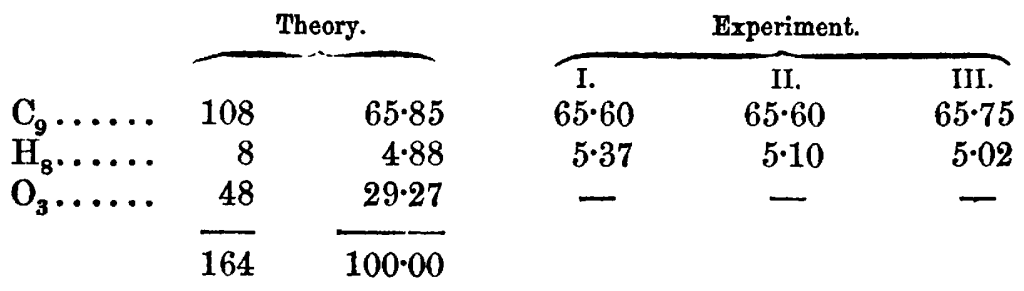


This substance represents the hydride of salicyl with an equivalent of hydrogen replaced by acetyl. The formation may be expressed thus:-

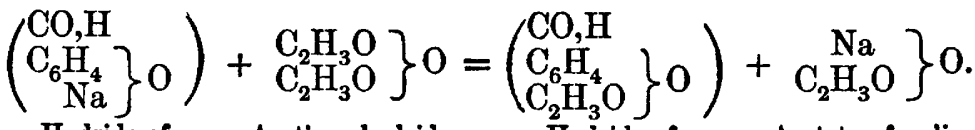

$$
\begin{aligned}
& \text { Hydride of Acetic anhydride. Hydride of Acetate of sodium. } \\
& \text { sodium salicyl. } \\
& \text { aceto-salicyl. }
\end{aligned}
$$

The hydride of aceto-salicyl melts at $37^{\circ} \mathrm{C}$, and solidifies upon cooling into a satiny crystalline mass. At about $253^{\circ} \mathrm{C}$. it boils, and distils with but little decomposition. It is extremely soluble in alcohol, from which it crystallises in fine silky needles. It is also very soluble in ether. The solutions do not give a purple reaction with ferric salts.

The hydride of aceto-salicyl is an aldehyde and combines with the bisulphites. It dissolves easily in the bisulphites of sodium and ammonium, and with the former forms a white pasty product; this, however, dissolves on standing, apparently owing to the decomposition of the hydride of aceto-salicyl.

This substance is also an acetate, and with alcoholic hydrate of potassium rapidly decomposes, with formation of acetate of potassium and hydride of potassium-salicyl (salicylite of potassium).

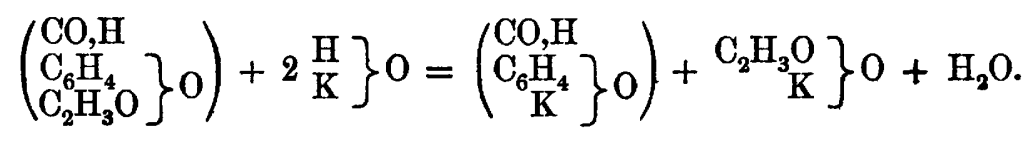

It is also decomposed into acetic acid and hydride of salicyl by boiling with water.

Hydride of Aceto-salicyl and Acetic Anhydride.

The hydride of aceto-salicyl and acetic anhydride, when heated together for three or four hours to $150^{\circ} \mathrm{C}$., in a sealed tube, yield a clear, slightly brown solution. On agitating this with a solution of carbonate of sodium to remove any excess of acetic anhydride, a crystalline body is obtained, which may be rendered pure by one or two crystallisations from 
alcohol. A combustion of this product gave the following numbers :-

2513 of substance gave

.5379 of $\mathrm{CO}_{2}$, and

$\cdot 1226$ of $\mathrm{H}_{2} \mathrm{O}$.

These numbers give percentage agreeing with the formula-

$$
\mathrm{C}_{13} \mathrm{H}_{14} \mathrm{O}_{6}=\mathrm{C}_{9} \mathrm{H}_{8} \mathrm{O}_{3}, \mathrm{C}_{4} \mathrm{H}_{6} \mathrm{O}_{3} \text {, }
$$

as the following comparisons will show :-

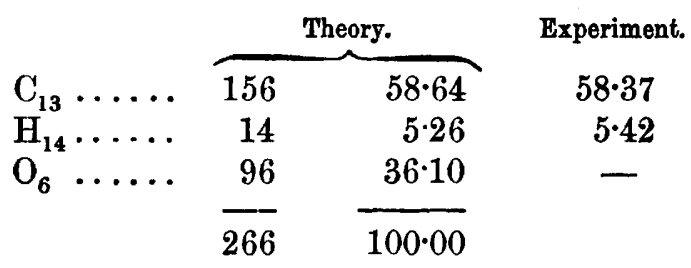

This substance is therefore a compound of the hydride of aceto-salicyl and acetic anhydride, corresponding to that obtained from the hydride of salicyl under the same circumstances-

$$
\underbrace{\left.\left(\begin{array}{l}
\mathrm{CO}, \mathrm{H} \\
\mathrm{C}_{6} \mathrm{H}_{4} \\
\mathrm{H}^{\prime}
\end{array}\right\} \mathrm{O}\right), \mathrm{C}_{4} \mathrm{H}_{6} \mathrm{O}_{3}}_{\text {Hydride of salicyl and acetic }}
$$
anhydride.

$$
\left.\left(\begin{array}{l}
\mathrm{CO}, \mathrm{H} \\
\mathrm{C}_{6} \mathrm{H}_{4} \\
\mathrm{C}_{2} \mathrm{H}_{3} \mathrm{O}
\end{array}\right\} \mathrm{O}\right), \mathrm{C}_{4} \mathrm{H}_{6} \mathrm{O}_{3} .
$$

Hydride of aceto-salicyl and acetic anhydride.

This compound melts at $100^{\circ}-101^{\circ} \mathrm{C}$. When distilled it decomposes, the products consisting chiefly of acetic anhydride and hydride of aceto-salicyl. When dissolved in acetic anhydride and the resulting solution exposed to the air, it is deposited in splendid brilliant oblique four-sided tables. It is easily soluble in hot, but only sparingly so in cold alcohol. It is also soluble in ether, tetrachloride of carbon, and benzole.

When it is heated in a sealed tube with water to $100^{\circ} \mathrm{C}$, for several hours, the water becomes acid, and if the temperature be maintained at $150^{\circ} \mathrm{C}$. for some time, it is entirely decomposed into acetic acid and hydride of salicyl. 


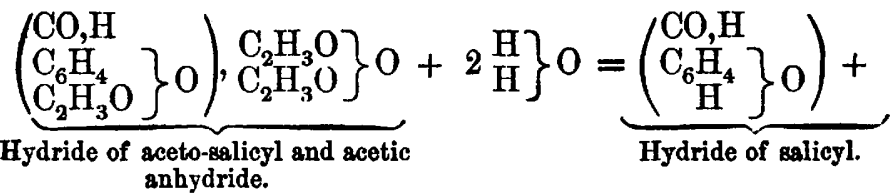

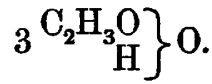

Formation of Coumarin from the Hydride of Aceto-Salicyl.

From the formation of the last-described substance it is evident that acetic anhydride does not decompose the hydride of aceto-salicyl, even at a temperature of $150^{\circ} \mathrm{C}$. I find also that no reaction takes place if these substances are distilled together, the acetic anhydride first coming over, and then the unchanged hydride of aceto-salicyl. Moreover, by the distillation of the combination of these two bodies, only traces of coumarin are produced. By what means then did I obtain coumarin by heating the hydride of sodium-salicyl and acetic anhydride, if the hydride of aceto-salicyl, being the first product of the reaction, is not dehydrated by acetic anhydride? Simply by what at first sight appeared to be of slight importance, viz., the presence of acetate of sodium as well as acetic anhydride.

On boiling a mixture of acetic anhydride, hydride of acetosalicyl, and acetate of sodium for a few minutes, and then agitating the product with water to remove acetate of sodium and the excess of acetic anhydride, the remaining product on being distilled, at first yields a little acetic acid and anhydride; the temperature then rises rather rapidly to $290.5^{\circ} \mathrm{C}$, when nearly pure coumarin distils over in considerable quantity.

The remarkable influence exercised by the presence of acetate of sodium in the foregoing experiments appears to be due to the presence of a sodium-compound analogous to the so-called anhydrous bracetate of potassium which Gerhardt obtained by heating acetate of potassium with acetic anhydride. That such a sodium-compound is produced I have proved by experiment;* but why it should be so much more

- Boiling acetic anhydride dissolves acetate of sodium, and the resulting solution on cooling deposits a new compound, crystallising in needles. This, when purified from adhering anhydride by pressure between bibulons paper, and 
powerful a reagent than the anhydride itself is difficult to understand.

It will be seen that the foregoing results corroborate the views regarding the formation of coumarin brought forward in my previous paper.

I have not as yet produced the hydrides of butyryl-and valyerl-salicyl, from which the new homologues of coumarin were doubtless produced, but hope to do so shortly. 